\title{
Creación de textos inéditos, una estrategia asertiva en el aula
}

\section{Creation of unpublished texts, an assertive strategy in the classroom}

Fecha de recepción: 6 de junio de 2017

Fecha de aprobación: 20 de noviembre de 2017

Yaneth Gómez Rojas* Patricia Torres Alarcón *** Merly Astrid Varón Bedoya *\%:

Artículo corto

\section{Resumen}

El propósito de esta investigación se enfocó en analizar, al interior del contexto escolar, falencias y debilidades presentadas por estudiantes de Grado Quinto de la Institución Educativa Politécnico Álvaro González Santana, Sogamoso, sedes Rosario y Manitas, cuyo objetivo refería la motivación de los estudiantes por la creación de textos inéditos que expresen la realidad de su contexto.

Esta propuesta incluyó una metodología cualitativa de tipo crítico-social, con una acción investigación, en donde los hallazgos muestran que los estudiantes, en su mayoría, presentan desidias en el momento de la creación textual, evidenciado en el bajo nivel de producción escrita, falta de cohesión, coherencia, construcción de frases y estructuración de ideas. Se pudo demostrar que, al momento de llevar a los estudiantes estrategias innovadoras, su creación de textos escritos fue enriquecedora, la denominada desidia pasaba a un segundo plano y se activó un interesante momento de creación.

Palabras clave: Enseñanza, motivación, aprendizaje, estrategia, producción escrita.
* Institución Educativa Politécnico Álvaro González Santana, Sogamoso Boyacá yanecitag@hotmail.com

** Institución Educativa Politécnico Álvaro González Santana, Sogamoso Boyacá torrespatricia2010@hotmail. com

*** Institución Educativa Politécnico Álvaro González Santana, Sogamoso Boyacá merlyastrid@gmail.com 


\section{Abstract}

The purpose of this research was to analyse, within the school context, flaws and weaknesses presented by fifth grade students of the Educational Institution "Álvaro González Santana", "Rosario" and "Manitas" branches whose objective was to encourage students on the creation of unpublished texts that express the reality of their context. This proposal included a qualitative methodology of socio-critical type, with a research action, where the findings show that the students, in their majority, present neglect at the moment of the textual creation, evidenced in the low level of written production, lack of cohesion, coherence, construction of sentences and structuring of ideas. It was possible to demonstrate that, when taking innovative strategies to the students, their creation of written texts was enriching, the so-called neglect went into the background and an interesting moment of creation was activated.

Keywords: Teaching, motivation, learning, strategy, written production. 


\section{Introducción}

El proyecto de investigación "Creación de textos inéditos, una estrategia asertiva en el aula" Buscó indagar al interior del Grado Quinto, por qué los estudiantes se muestran inseguros, apáticos y desmotivados frente al acto de crear textos, expresar sus emociones, sentimientos, pensamientos, y concatenar ideas de forma ordenada y coherente.

En tal sentido, se hizo necesario, desde la práctica directa y análisis de la situación, formular la pregunta base de esta investigación, que se plantea así: ¿Qué estrategias metodológicas se pueden implementar en el aula para fomentar la creación de textos escritos en los estudiantes de grado $5^{\circ}$ ? Es así; como toma cuerpo el objetivo de potenciar las habilidades comunicativas, en el nivel de producción escrita, con el fin de incentivar la creación de textos en este grupo de estudiantes.

Dentro de las fases del proyecto de investigación, se realizó un diagnóstico in situ, el cual conllevó a identificar dificultades presentadas en los estudiantes en la creación de textos, con quienes, posteriormente se utilizaron estrategias metodológicas que permitieran y favorecieran la producción escrita como base de intervención pedagógica de la propuesta, para evaluar la efectividad de las didácticas aplicadas en el aula y así obtener un resultado de participación activa de la familia y contexto próximo donde el estudiante evidenciará sus inéditos en publicaciones cercanas a su contexto.
Es importante mencionar que, para esta investigación se entiende la escritura como un proceso constante de aprendizaje, a través del cual se reflexiona en torno de conceptos propios del lenguaje, parafraseando a Joseph M. Espinas, en el Prólogo de texto la Cocina de la Escritura de Daniel Cassany (1993), cuando menciona que:

La mayoría de adolescentes se sienten muy inseguros cuando tienen que explicar algo e incluso aceptan su incapacidad. Esto no es bueno. Hay que darse cuenta que redactar correctamente - lo cual no es un indicio de sensibilidad literaria es ante todo un problema "técnico" y que debe resolverse a tiempo para que no se convierta en un problema psicológico p. 145).

Es por esto que, en la actualidad, el escribir se convierte en una dificultad latente, masiva y generalizada en las aulas de clase. El acto comunicativo de escribir es un elemento fundamental para la transmisión y generación de conocimientos en los procesos de enseñanza-aprendizaje, tanto en docentes como en estudiantes, para hacer uso de esta habilidad en el aula e interactuar con el contexto en el cual se desarrolla cada momento del acto educativo, enfocado al desarrollo de habilidades comunicativas.

Otro referente es tomado desde la perspectiva de Solé, (1987), para quien "Las habilidades comunicativas, son las formas en que el ser humano utiliza la lengua para diferentes propósitos: comunicarse, aprender y pensar" Desarrollo de habilidades comunicativas, pág..7, párr.6. Estas son
La mayoría de adolescentes se sienten muy inseguros cuando tienen que explicar algo e incluso aceptan su incapacidad. Esto no es bueno. Hay que darse cuenta que redactar correctamente - lo cual no es un indicio de sensibilidad literaria es ante todo un problema "técnico" y que debe resolverse a tiempo para que no se convierta en un problema psicológico p. 145). 
fundamentalmente cuatro: escuchar, hablar, leer y escribir. Las habilidades de escuchar y hablar corresponden al nivel oral de la lengua; y las de leer y escribir, al nivel escrito; clasificándose en habilidades receptivas (escuchar y leer), y habilidades productivas (hablar y escribir). Por lo tanto, se considera que estas deben desarrollarse de manera integral y con la participación activa de las niñas y los niños.

\section{Reconstructores de lo construido}

Del mismo modo, se consideró importante para la investigación, tener en cuenta este cúmulo de capacidades que llevan al niño al uso eficiente de la lengua, la participación e interacción entre las personas cuando se tienen objetivos de comunicación específicos, delimitados, y conscientes los cuales permitieron evidenciar procesos comunicativos personales en los estudiantes.

Conocer los aportes de algunos autores frente a las habilidades comunicativas para precisar algunos conceptos teóricos de esta temática a tratar, es relevante; puesto que, las habilidades comunicativas inciden directamente en la creación y producción textual la cual fue el objetivo principal de este equipo de investigación. Para lo cual se analizarán aspectos conceptuales abordados por Solé, (1987) y Carlino (2006).

La forma escrita del lenguaje es parte de una cultura humana compleja, sus formas están limitadas por valores y costumbres de la cultura. Por lo tanto, se incluyó como eje fundamental a las familias de los educandos intervenidos para que apoyaran este proceso educativo, el cual permitió conocer modelos y teorías predominantes en la producción de textos, los cuales consintieron una motivación constante de los estudiantes, invitándolos a escribir partiendo de sus experiencias cotidianas ya fuesen de tipo personal, familiar o social, vinculando en este proceso factores que influyen en su propio contexto como culturales, sociales, afectivos, cognitivos, metacognitivos, discursivos, pragmáticos, entre otros, que intervienen en el proceso de producción textual.

Organización General del Modelo

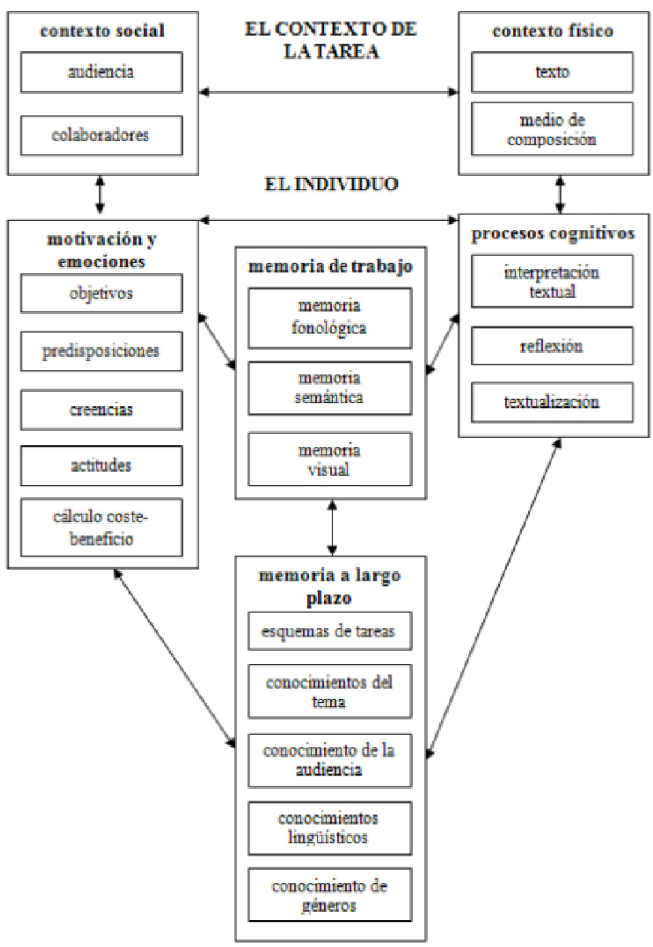

Figura 1. Modelo cognitivo de composición escrita Fuente: Hayes; (1996). 
Se debe resaltar que, este tipo de investigaciones han venido inundando los espacios escolares, pero todos tienen valores distintos, desde la diversidad situacional en el que cada uno de ellos se va ocupando; es por esto; que, la reflexión acerca de la composición escrita ha generado modelos que enfatizan ya sea en productos, textos de diversas características y finalidades, o en los procesos que se ponen en juego hasta elaborarlos y producirlos.

\section{Del diálogo de saberes o de la discusión teórica}

En el contexto colombiano, el Ministerio de Educación Nacional (MEN) define el escribir, como una competencia comunicativa que se realiza en situaciones concretas, y con propósitos claros. Eso quiere decir que, en el aula, en la escuela producimos textos escritos, cuando tenemos la necesidad de comunicarnos, relacionarnos con otros, expresar nuestras ideas y sentimientos, crear, investigar o aprender. Es aquí cuando la investigación aborda el lenguaje también como un proceso de creación, de transformación y de libertad (Samacá, 2016; Mariño, Pulido \& Morales, 2016).

No obstante, resulta complejo abordar esta fase investigativa de la producción de texto como estrategia didáctica pedagógica, sin enfatizar en qué consiste el proceso de la comunicación, y para esto el estudio se basa en los aportes de algunos expertos en el tema que han contribuido en el proceso desde la pedagogía y el aprendizaje.
De ahí, la importancia de que se aborde la mirada de Grimson (2006), quien establece que: "La comunicación es un todo integrado, donde se concibe imposible no comunicarse; es decir, nos encontramos frente a un ser comunicativo en esencia". Desarrollo de habilidades comunicativas, pág., 7, párr.6. Desde ese postulado, se puede establecer que los estudiantes del Grado Quinto de la Institución Educativa Politécnico Álvaro González Santana, Sogamoso, sedes Rosario y Manitas, se relacionan y expresan entre ellos de manera natural, pero no es así cuando el docente, pretende la comunicación a través de esa frescura comunicativa que sí se da entre ellos. De ahí, la importancia de que las estrategias desarrolladas; implementen el trabajo, los lenguajes y el desparpajo con que los estudiantes abordan sus cotidianas comunicaciones.

Cada estudiante escribe según sus motivaciones e intereses, el proceso de escribir de forma inédita es tan personal y emocional, que lleva implícito el ser, y no permite protagonistas ajenos a su autor. Es por esto que, los docentes deben estar atentos a conocer a sus estudiantes dentro y fuera del aula, para a la par, desarrollar habilidades que les permitan conocerse y conocer el mundo que les rodea. Reconociendo que hoy día los estudiantes se interesan más por los medios audiovisuales y tecnológicos, que, por los manuscritos, desconociendo el valor de estos y alejándose de la creación de textos en físico. El maestro también debe saber adaptar sus saberes a estas nuevas realidades.
En el contexto colombiano, el Ministerio de Educación Nacional (MEN) define el escribir, como una competencia comunicativa que se realiza en situaciones concretas, y con propósitos 
El lenguaje escrito consiste en un sistema de signos que designan los sonidos y las palabras del lenguaje hablado y que, a su vez, son signos de relaciones y entidades reales. Gradualmente este vínculo intermedio que es el lenguaje hablado desaparece, y el lenguaje escrito se transforma en un sistema de signos que simbolizan directamente las relaciones y entidades entre ellos.
En ese sentido, esta investigación dio gran relevancia a la creación de textos, como instrumento de interpretación de las realidades de los niños, tal como los establece Kaplún (1998), considerando que:

El niño tiene que escribir para ser leído, el niño que comprueba la utilidad de su labor, que puede entregarse no sólo escolar sino también social y humanamente, siente liberarse en su interior una imperiosa necesidad de actuar, buscar y crear a medida que escriben y ven sus escritos publicados y leídos, se va despertando su curiosidad, su apetencia de saber más, de investigar más, de conocer más (p..207).

En consecuencia, el niño que escribe se libera de sus propios temores, es capaz de ser leído y conocido sin miedo al ridículo, creando autonomía personal y seguridad en sus textos. Ahora bien, resaltando esta tesis, la investigación se apoyó en el trabajo desarrollado por Peñarrieta Bedoya (2010), en donde relaciona que "aprender significativamente supone pues modificar los esquemas de conocimiento del sujeto, reestructurar, revisar, ampliar, enriquecer las estructuras cognitivas organizadas existentes"(p..54), dejando de manifiesto que no solo es hacer que el niño cree textos, sino que los textos signifiquen para el proceso de enseñanza-aprendizaje, un resultado favorable en la comunidad estudiantil y docente.

Pero esto no puede ser solo implementando estrategias metódicas que lleven al estudiante a algo ideal, para esto es preciso que el maestro para enseñar a expresar, entender $y$ comprender según su contexto, sea él un creador de textos en donde su comunicación es fuente de vida para otros creadores de textos: sus estudiantes.

Se tiende a considerar el lenguaje escrito como una variable relacionada con el contexto social, ya que es el contexto lo que forma y conforma el sistema de escritura. Vygotsky (1979), define el lenguaje escrito como un sistema simbólico de segundo grado que, poco a poco, se va convirtiendo en un simbolismo directo. Esto significa, según sus propias palabras, que:

El lenguaje escrito consiste en un sistema de signos que designan los sonidos y las palabras del lenguaje hablado y que, a su vez, son signos de relaciones y entidades reales. Gradualmente este vínculo intermedio que es el lenguaje hablado desaparece, y el lenguaje escrito se transforma en un sistema de signos que simbolizan directamente las relaciones y entidades entre ellos. (p. 75).

Es entonces cuando se define que el lenguaje escrito prevalece en el tiempo y por encima del lenguaje hablado, gracias a sus códigos escritos y lingüísticos que favorecen la autenticidad del texto escrito, mientras que el texto hablado se compone de varios agentes que lo modifican.

En el trabajo desarrollado por el estudiante que aparece en la Figura 2 , se logró identificar que, con un instrumento de creación de textos, se podía acceder a información que con las tradicionales herramientas no se 


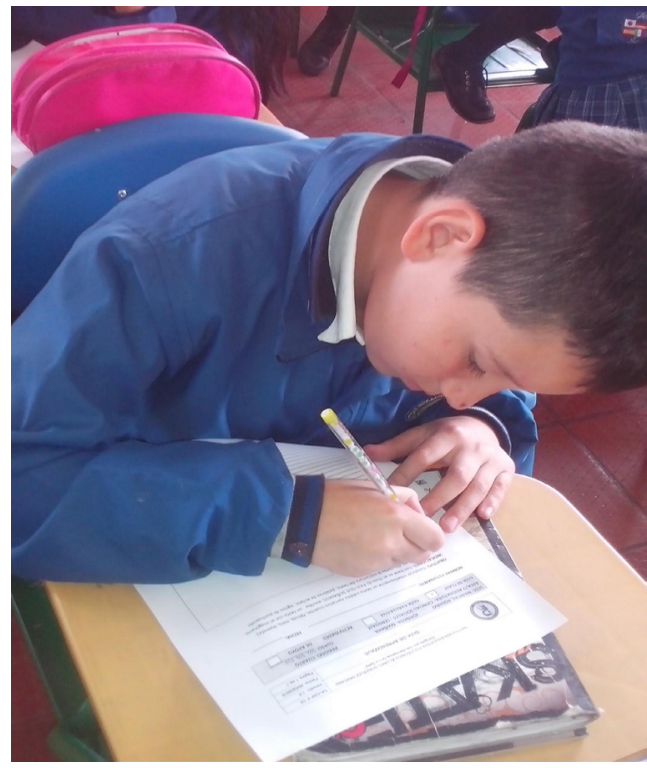

Figura 2. Desarrollo de talleres de producción escrita.

Fuente: archivo fotográfico equipo investigador, (2016).

lograba. Es decir, que el estudiante no se sentía perseguido por un trabajo que el profesor plantea. Tal como lo establecía Kaplún anteriormente, el estudiante quería ser leído desde sus realidades, desde sus inquietudes y sus lenguajes.

\section{De la metodología y los resultados}

El paradigma adoptado en esta investigación, es el cualitativo con enfoque crítico-social basado en la investigación acción, que enmarca al docente como un facilitador de habilidades y estimulador de procesos, que busca ser un investigador de su propia práctica en el aula, convirtiéndola en un escenario de transformación.

La institución educativa intervenida, Politécnico Álvaro González Santana, La Salle, cuenta con una población de
4200 estudiantes distribuidos en cinco (5) sedes, una (1) de básica secundaria y media técnica, cuatro (4) sedes de preescolar y básica primaria; y para la presente investigación se trabajó en dos sedes: Rosario y Manitas tomando como muestra 108 estudiantes del grado quinto de la totalidad de los cursos: 502 , 509 y 510 .

El contexto próximo que nos atañe con los estudiantes de la Institución Educativa Politécnico Álvaro González Santana del municipio de Sogamoso, sedes Rosario y Manitas, grado $5^{\circ}$, es la indiferencia, displicencia y desinterés por la práctica de una correcta escritura; autónoma, creativa y significativa, evidenciando apatía $y$, falta de acompañamiento familiar en el proceso de producción escrita y creación de textos inéditos.

Se indagó que, sobre este proceso escritor, la institución educativa en mención aún no ha liderado estrategias claras, pertinentes, o políticas claras institucionales que permitan abordar estas debilidades globales como área; sin embargo, no se desconoce que en cada práctica de aula los docentes propenden de manera particular por este fin, pero en torno al tema, actualmente, no hay antecedentes o análisis de casos específicos de la institución en esta problemática escolar.

Con base en la descripción anterior, fue necesario analizar los índices sintéticos de calidad de la Institución Educativa Politécnico Álvaro González Santana de la Salle, del municipio de Sogamoso, y los resultados de las pruebas externas saber grado $5^{\circ}$ de los
El paradigma adoptado en esta investigación, es el cualitativo con enfoque crítico-social basado en la investigación acción, que enmarca al docente como un facilitador de habilidades y estimulador de procesos 


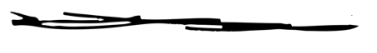

Las técnicas y métodos utilizados en esta investigación, fueron de la observación directa y participante en el aula, por medio de la aplicación de talleres pedagógicos años 2014 y 2015, los cuales arrojan resultados con tendencia media y baja en el área de lenguaje durante los dos últimos años respectivamente, lo cual permitió detallar cómo está posicionada la institución educativa a nivel local, regional y nacional en la actualidad.

Las técnicas y métodos utilizados en esta investigación, fueron de la observación directa y participante en el aula, por medio de la aplicación de talleres pedagógicos; primero, diagnósticos que buscaron producción y creación de textos inéditos que aportaron con determinación real en el lugar y contexto a intervenir.

Posteriormente, los talleres pedagógicos pasaron de ser diagnósticos a ser de formación en la producción y creación de textos inéditos. Se hace referencia a la etapa diagnóstica en la cual fue pertinente determinar que los estudiantes muestran pereza, desidia apatía al escribir y es en el primer taller aplicado que esta problemática se deja percibir claramente, los estudiantes al observar una imagen referida a la contaminación, solamente escriben palabras de lo que hay en la imagen refiriéndose a nombres de objetos o sustantivos, sin ir más allá de escribir descriptivamente lo que observan, porque les cuesta trabajo el proceso de organizar ideas, tienen temor a equivocarse o sencillamente porque no desean hacer más de lo que implique la guía propuesta aludiendo al mínimo esfuerzo. Abordando progresivamente la problemática, se planea la organización de nuevos talleres o guías pedagógicas encaminadas a disminuir o contribuir a la mejora de estas falencias $y$ debilidades percibidas; como lo fue la creación de fábulas y autobiografías de los estudiantes, donde tuvieron la oportunidad de escribir textos titulados "Mi historia de vida" o "así soy yo" con un fuerte motivacional para producir sus historias. Otra estrategia de creación textual tuvo lugar con la interpretación de cóomics mudos que llevaban implícita una historia que el estudiante podía recrear.

Otro instrumento pedagógico utilizado fue el uso del "cuaderno viajero", como una herramienta eficaz que recogió historias de cada familia, escritas por estudiantes intervenidos y narradas por sus familiares casa a casa, con el objeto de rescatar historias y vincular el grupo familiar en el proceso incentivando en los niños su espíritu narrativo. Con esta poderosa herramienta, se pudo cualificar la investigación y a la vez despertar en los estudiantes su espíritu narrativo de historias personales $y$ familiares, que enriquecieron el acto de la escritura inédita y mostraron que todos podemos y tenemos relatos por contar, historias por crear y cuentos por imaginar. Fue estimulante para el grupo investigador ver la participación activa de la familia en el proceso de llevar a diario el cuaderno a sus casas, ellos como familia pudieron detallar que es en la familia donde se recrean situaciones que perduran en el recuerdo para toda la vida; a la vez que se despertaba la curiosidad por leer las aventuras que sus compañeros habían escrito e ilustrado en días anteriores, y al otro día llegaban a verificar si en realidad esta narración la habían experimentado, buscando que desde la oralidad les contaran en vivo la 
historia ya narrada en forma escrita.

En su momento, la docente revisó corrigió la redacción y ortografía, y motivó e incentivó para que el niño transcribiera correctamente su escrito en otra hoja denotando por sí mismo las dificultades presentadas en cuanto a ortografía, uso adecuado de signos de puntuación, coherencia y cohesión en la construcción de ideas, frases y textos narrativos correctamente escritos. Fomentando así el deseo de escribir, tomándolo como hábito, herramienta o técnica escolar.

Como se puede observar, es un proceso que se va desarrollando y el niño va creando conciencia de la importancia

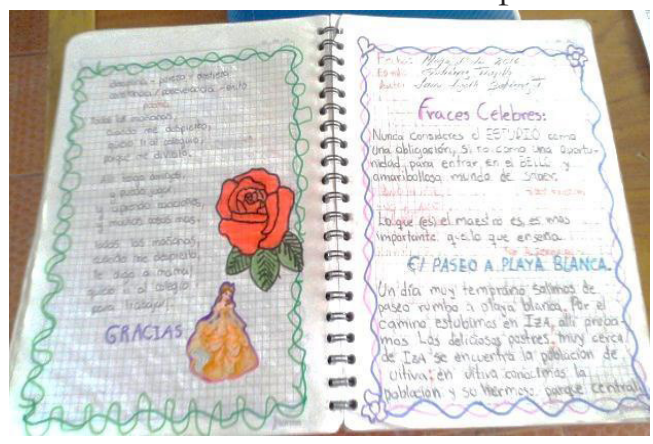

Figura 3. Estrategia cuaderno viajero.

Fuente: autoría del equipo investigador.

Esta investigación permite detallar que escribir es un proceso que implica motivación, disciplina y dedicación, que con la aplicación de talleres pedagógicos y ejercicios lúdicos dirigidos no es suficiente; sin embargo, se nota el avance, aunque se tiene; claro que es la práctica la que con el correr de los años permitirá demostrar y evaluar la capacidad y habilidad para escribir. De ahí que, se pretende motivar a los docentes para que en las aulas se siembre la semilla del descubrir el mundo escribiendo y de expresar ideas, emociones y sentimientos de manera sencilla, clara y precisa.

Dando cumplimiento a las metas planeadas por las investigadoras y luego de analizar de manera juiciosa y responsable el proceso escritor de los estudiantes, se organizan los textos que por niño merecen ser publicados, dando así respuesta a uno de los objetivos específicos de la propuesta, como es el de publicar textos narrativos inéditos con el objeto de continuar despertando en el niño el deseo de escribir y plasmar de manera contextualizada, creativa, fluida e inédita sus anécdotas o historias que merecen ser contadas dejándose ver como escritores en potencia idealizados con el tiempo como grandes escritores.

Se aplicó también una encuesta a los actores educativos: directivos docentes, docentes y estudiantes; la cual fue un instrumento eficaz de recolección de información, que permitió la triangulación entre estos estamentos y el análisis de variables sobre producción textual. Esta nos orientó en la triangulación de datos, como la confrontación de diferentes fuentes de datos en un estudio. La triangulación se produce cuando existe concordancia o discrepancia entre estas fuentes. Además, se pueden triangular informantes/personas, tiempos y espacios/contextos, después de la triangulación de datos de información se pudo analizar, argumentar y definir la producción textual como una falencia sentida en las aulas de clase
Esta investigación permite detallar que escribir es un proceso que implica motivación, disciplina y dedicación, que con la aplicación de talleres pedagógicos y ejercicios lúdicos dirigidos no es suficiente 
de la Institución Educativa Politécnico Álvaro González Santana y significativa en los grados $5^{\circ}$, que motiva a ser intervenida de manera asertiva en los estudiantes y en las estrategias didácticas utilizadas por los docentes, a la vez, en las políticas institucionales de directivos docentes de las sedes Rosario y Manitas, grados 502, 509 y 510 .

\section{Resultados}

Dentro del desarrollo metodológico del proyecto, se logró detectar que los estudiantes avanzan en su proceso de creación de textos escritos, siempre y cuando se dé libertad a su inquietud, dando valor a cada persona desde su originalidad, sus contextos y sus realidades. El maestro puede llevar muchas estrategias, pero si no da el valor a sentir del estudiante se puede concluir que todas serán infértiles.

Los resultados muestran que la producción textual se ha convertido en un reto autónomo para los estudiantes que manifiestan constantemente el deseo por querer construir a partir de sus textos $y$, por ende, se inquietan para dar a conocer sus pensamientos a través de textos, que naturalmente deben ser leídos, como símbolo de respeto por los pensamientos de los estudiantes.

Al involucrar a los estudiantes en procesos escriturales autónomos $\mathrm{y}$ activos, se logra favorecer y contribuir a mejorar estándares de calidad educativa desde la narrativa reflejados en el índice sintético de calidad de la Institución Educativa. Se proyectan estudiantes con rendimientos académicos satisfactorios y, por lo tanto, se apunta a subir los promedios en pruebas internas y externas, a la vez en busca de estudiantes gestores, participativos, críticos e inquietos por un legado $y$ rescate de producción textual en su contexto más cercano.

Cabe mencionar que, no todos los estudiantes llegarán a ser creadores de textos con estas estrategias, pues, la disposición de todos entra en juego, esta no es una receta que funcione infaliblemente, es todo lo contrario, debe ser interpretada para los diferentes momentos de los estudiantes y los ritmos institucionales, es decir, en etapas de evaluaciones, con dificultad se conseguirán en el aula, pero quizá sí en sus estados de intimidad consigo mismos.

\section{Conclusiones}

Evidentemente, los estudiantes reflejan que existe temor para plasmar escritos; hay rechazo, apatía y desidia por querer escribir sus propios textos; este proceso requiere seguir una secuencia metodológica, lúdica y didáctica sugerida y orientada por los docentes, quienes tienen la tarea de motivar e incentivar constantemente al estudiante a desarrollar y potenciar sus habilidades comunicativas, en este caso la de escribir.

Al analizar esta propuesta, la de implementar la producción del texto como una estrategia asertiva en el aula, permite detallar que escribir es un proceso que implica motivación, disciplina y dedicación, que con la aplicación de talleres pedagógicos y ejercicios lúdicos dirigidos no es 
suficiente. Sin embargo, se nota el avance, aunque se tiene claro que es la práctica la que con el correr de los años permitirá demostrar y evaluar la capacidad y habilidad para escribir.

Partiendo de que el arte de escribir es un proceso, es importante despertar en los estudiantes desde los primeros años escolares, el deseo de escribir abordando y vinculando las diferentes habilidades detalladas en los procesos de pensamiento complejo, como es la de analizar, clasificar, comparar, inferir, recordar, interpretar, contextualizar, planear, pensar, organizar, mirar, borrar, corregir, subrayar, entre otras.

Es preciso para ello, convertir el aula y otros ambientes de aprendizaje en un espacio abierto de reflexión, debate, cuestionamiento y contrastación de las diferentes perspectivas en torno a problemas de la vida, a los específicos del área de formación como personas y de aquellos propios de la sociedad, donde el escritor se convierte en un sujeto capaz de cambiar su postura hacia un agente consciente de interpretación, creación y transformación de la vida y la cultura; preparando así a los estudiantes para participar con plena libertad y autonomía en el quehacer de la ciudadanía.

La experiencia de haber trabajado esta propuesta en el aula, para las investigadoras, fue muy significativa, $\mathrm{y}$ los resultados hasta el momento han sido satisfactorios. También, a nosotras nos permitió tener un pensamiento más reflexivo, crítico, analítico, autónomo y responsable acerca de nuestro quehacer cotidiano dentro y fuera del aula.

"A escribir se aprende escribiendo.

Es un trabajo, se aprende baciéndolo, como cualquier otra disciplina"

(Hebe Uhart, (2010). Relatos Reunidos.

\section{Referencias}

Carlino, P. (2006). Escribir, Leer y aprender. Madrid, España: Fondo de la Cultura Económica

Cassany, D. (1993). La cocina de la escritura. Barcelona, España: Anagrama

Grimson, A. (2006). Relatos de la diferencia y la Igualdad. Brasilia: Felfac

HAYES, J. (1996). Un nuevo marco para la comprensión de lo cognitivo y lo emocional en la escritura.

Mariño Díaz, L., Pulido Cortés, O., \& Morales Mora, L. (2016). Actitud filosófica, infancia y formación de maestros. Praxis \& Saber, 7(15), 81-101. https://doi.org/10.19053/22160159.v7.n15.2016.5724

Mendoza Alfredo. (2015) Desarrollo de habilidades lingüísticas. Pág. 07, párr. 6.

La experiencia de haber trabajado esta propuesta en el aula, para las investigadoras, fue muy significativa, y los resultados hasta el momento han sido satisfactorios. 
La armonía y el equilibrio familiar son la fuente permanente de armonía y seguridad en la vida de los hijos. De los padres, principalmente, aprende los "papeles" que tienen que desarrollar en la vida adulta, es más, el propio ambiente familiar es el contexto adecuado en el que el niño ensaya y experimenta los roles que tendrá que realizar cuando sea adulto.

New Jersey: Erlbaum.

KaplúN, M. (1998). Una pedagogía de la comunicación. Madrid: Ediciones de la Torre.

Peñarrieta, L. (2010). Desarrollo de habilidades comunicativas básicas en niños de 9 a 13 años a través de la radio. Revista de Investigación en comunicación y desarrollo. 1 (1), 53-61.

Samacá Bohórquez, I. (2016). El espíritu científico en la primera infancia. Praxis \& Saber, 7(13), 89 - 106. https://doi.org/10.19053/22160159.4167

Solé, I. (1987). Estrategias de lectura y escritura. Barcelona, España: Grao

Uhart Hebe (2010) Relatos Reunidos, Argentina: Alfaguara.

VyGOTSKY, L. (1979). Historia del desarrollo de las funciones psicológicas. La Habana. Científico técnico. 